\title{
NONLINEAR TECHNIQUES FOR LINEAR OSCILLATION PROBLEMS $\left({ }^{1}\right)$
}

BY

\section{ZEEV NEHARI}

\begin{abstract}
It is shown that for differential equations of the form $y^{(n)}+$ $p y=0$ there exist associated sets of systems of nonlinear equations which play a role similar to that of the ordinary Riccati equation in the case $n=2$. In particular, the existence of continuous solutions of the nonlinear system is equivalent to the absence of certain types of oscillatory solutions of the linear equation. If $p$ is of constant sign, the coefficients of the "Riccati systems" are all nonnegative, and the resulting positivity and monotonicity properties make it possible to obtain explicit oscillation criteria for the original equation.
\end{abstract}

1. One of the classical techniques employed in the discussion of oscillation problems for linear second-order differential equations consists in replacing the study of the original equation by that of the equivalent Riccati equation. While the linearity of the original problem is sacrificed in this process, the formal structure of the Riccati equation permits-indeed invites-analytic manipulations which were not possible, or at least not apparent, in the original formulation. In fact, as pointed out in E. Hille's fundamental paper on the subject [4], this seeming detour via the Riccati equation may in certain respects be regarded as the "natural" approach to the linear oscillation problem.

In a recent paper [14] it was shown that a similar approach is available for differential equations of the form $x^{\prime}=A x$, where $x$ is an $n$-dimensional vector function and $A$ a continuous $n \times n$ matrix. There exists a "Riccati system", i.e., a system of nonlinear differential equations which is equivalent to the original equation if and only if the latter is nonoscillatory in a certain sense, and this connection between the linear and nonlinear systems leads to effective ways of attacking various oscillation problems associated with the original equation. These Riccati systems, incidentally, are not related to the "matrix Riccati equations" [15], [2] which are used in the treatment of second-order vector-matrix equations and which represent a natural extension of the classical formalism.

Since linear $n$th order differential equations are special "cases of the equation $x^{\prime}=A x$, they can be treated by the methods described in [14]. In fact, this treatment can be considerably simplified because of the particular character of the

Received by the editors July 19, 1974.

AMS (MOS) subject classifications (1970). Primary 34C10.

$\left.{ }^{1}\right)$ Research supported by the National Science Foundation under Grant GP 23112.

Copyright $\odot 1975$, American Mathematical Society 
matrices $A$ associated with such equations. In [14], some of these simplifications are carried out up to a certain point, but the difficulties mount and it rapidly becomes clear that the proper way of treating an equation of simple build like

$$
u^{(n)}+q(t) u=0
$$

is not to regard it as a special case of $x^{\prime}=A x$, but to take advantage of the special form of the equation. The Riccati system (or rather, as will be seen, the various Riccati systems) associated with equation (1.1) can be easily obtained, and these systems can then be used to derive a variety of explicit, and easily applied, oscillation criteria for the equation.

2. We begin with some general remarks concerning the oscillatory character of equations of the form (1.1). The equation is said to be nonoscillatory on $[0, \infty)$ if none of its solutions has an infinite number of zeros in this interval (unless the contrary is stated, the word "solution" will be used as an abbreviation for "nontrivial solution"). If the equation has a solution with more than $n-1$ zeros (counting multiplicities) on $[0, \infty)$, then there exists a point $\zeta \in(0, \infty)$ such that no solution of $(1.1)$ has more than $n-1$ zeros on $[0, \zeta]$, except for one particular solution which has zeros of order $k$ and $n-k(1 \leqslant k \leqslant n-1)$ at 0 and $\zeta$, respectively, and does not vanish in $(0, \zeta)[9] . \zeta$ is referred to as the point conjugate to 0 . If $n>4$, it is in general difficult to predict the value of $k$, but if the coefficient of the equation is of constant sign there are some values of $k$ which are excluded a priori. For an equation of the form

$$
u^{(n)}+p u=0, \quad p>0,
$$

$n-k$ must be odd, and for the equation

$$
u^{(n)}-p u=0, \quad p>0,
$$

$n-k$ must be even [9], [13]. In deriving criteria for the existence or nonexistence of conjugate points, equations (2.1) and (2.2) may therefore be replaced by the single equation

$$
u^{(n)}-(-1)^{n-k} p u=0, \quad p>0 .
$$

An equation which does not have a conjugate point on $[0, b)$-or, equivalently, an equation which does not have a solution with more than $n-1$ zeros on $[0, b)-$ is said to be disconjugate on $[0, b)$. If the interval of disconjugacy is $[0, \infty)$, we shall say, briefly, that the equation is disconjugate. Evidently, disconjugacy implies nonoscillation. For $n=2,3,4$, a nonoscillatory equation of the form (2.1) or (2.2) is ultimately disconjugate, i.e., there exists a $t_{0}>0$ which has no conjugate point in $\left(t_{0}, \infty\right)$ [3], [5]. Whether this is true for $n>4$ is an open question. 
When it is necessary to indicate the multiplicities of the zeros of the solution associated with the conjugate point we shall write $\zeta_{k, n-k}$ instead of $\zeta$, and we shall refer to it as a "( $k, n-k)$-conjugate point" of 0 [9]. This concept may be extended to points $\zeta$ which are not conjugate points in the original sense, but are defined as the smallest $\zeta$ for which there exists a solution with zeros of order $k$ and $n-k$ at 0 and $\zeta$, respectively, where $k$ may be any number in $[1, n-1]$ which has the correct parity.

Together with the $(k, n-k)$-conjugate point, we shall consider the closely allied concept of the $(k, n-k)$-focal point $\eta=\eta_{k, n-k}$ (associated with the point 0 ), defined as the smallest positive number $\eta$ such that (2.1) or (2.2) has a solution for which

$$
\begin{aligned}
u(0) & =u^{\prime}(0)=\ldots=u^{(k-1)}(0)=u^{(k)}(\eta) \\
& =u^{(k+1)}(\eta)=\ldots=u^{(n-1)}(\eta)=0 .
\end{aligned}
$$

If the equation does not possess a $(k, n-k)$-focal point in $(0, b)$, it will be said to be $(k, n-k)$-disfocal on $[0, b)$; the statement that the equation is disfocal on $[0, b)$ will mean that it is $(k, n-k)$-disfocal on $[0, b)$ for all $k \in[1, n-1]$. If $b=\infty$, reference to the interval of disfocality will be omitted. The possible values of $k$ for focal points are the same as for conjugate points: for equation (2.1), $n-k$ must be odd, and for equation (2.2) $n-k$ must be even [13]. As before it is therefore sufficient to consider equation (2.3).

The methods which will be used in the following sections apply to focal points rather than to conjugate points. If it is desired to convert results concerning the presence or absence of focal points into statements about conjugate points-and thus, ultimately, about the zeros of solutions of the equations under consideration-it is necessary to have some information concerning the relations between conjugate and focal points. For $n=2,3,4$, and the interval $[0, \infty)$, the situation is very simple: The equation is disconjugate if and only if it is disfocal [3], [5]. This statement may well be true for $n>4$ but, in the general case, all we shall show here is that disfocality implies disconjugacy.

THEOREM 2.1. If equation (2.1) or (2.2) is disfocal, it is also disconjugate. More precisely, if equation (2.3) has a conjugate point $\zeta_{k, n-k}$ of 0 , it also has a focal point $\eta_{k^{\prime}, n-k^{\prime}}$ such that $0<\eta_{k^{\prime}, n-k^{\prime}}<\zeta_{k, n-k}, k^{\prime} \geqslant k$.

While we cannot prove the converse of this statement, we have the following two partial results.

THEOREM 2.2. If equation (2.1) is disconjugate, then it is at least $(n-1,1)$ disfocal. More precisely, if the equation has a focal point $\eta_{n-1,1}$, then it also has a conjugate point $\zeta_{n-1,1} \in\left(\eta_{n-1,1}, \infty\right)$. 
THEOREM 2.3. If equation (2.2), for odd $n$, is disconjugate, then it is at least $(1, n-1)$-disfocal.

Theorem 2.3 is a direct consequence of Theorem 2.2. The $(k, n-k)$-conjugate point of $(2.1)$ or $(2.3)$ coincides with the $(n-k, k)$-conjugate point of the adjoint equation (cf. [17, p. 169]), and a similar relation holds for focal points [13]. Since, for odd $n, u^{(n)}+p u=0$ is the adjoint of $u^{(n)}-p u=0$, an application of Theorem 2.2 establishes the assertion.

We first prove Theorem 2.1. If a solution $u$ of $(2.3)$ has $n$ zeros in $[0, b)$ and vanishes at 0 then, by Rolle's theorem, there exist points $b_{1}, \ldots, b_{n-1}$ in $[0, b)$ such that $u^{(\nu)}\left(b_{\nu}\right)=0, \nu=1, \ldots, n-1$. Moreover, these points may be chosen in such a way that $0 \leqslant b_{1} \leqslant b_{2} \leqslant \ldots \leqslant b_{n-1} \leqslant b$ and $u^{(v)}(t) \neq 0$ in $\left[0, b_{\nu}\right)$ if $b_{\nu}>0$. In particular, if $u$ is the solution associated with $\zeta_{k, n-k}, u$ has a zero of order $k$ at 0 and there exist points $b_{k}, \ldots, b_{n-1}$ such that $0<b_{k} \leqslant$ $b_{k+1} \leqslant \ldots \leqslant b_{n-1}<\zeta_{k, n-k}, u^{(\nu)}\left(b_{\nu}\right)=0, \nu=k, \ldots, n-1$, and $u^{(\nu)}\left(b_{\nu}\right)$ $\neq 0$ on $\left[0, b_{\nu}\right)$. We now pose the extremal problem $b_{n-1}=\min$, if $u$ ranges over all solutions of (2.3) which have these properties and which are, say, normalized by the condition $[u(0)]^{2}+\left[u^{\prime}(0)\right]^{2}+\ldots+\left[u^{(n-1)}(0)\right]^{2}=1$ (in order to exclude the trivial solution). The existence of a solution $v$ of (2.3) with this extremal property is elementary, and clearly $v$ has a zero of order $k^{\prime}\left(k \leqslant k^{\prime} \leqslant n-1\right)$ at 0 . Theorem 2.1 will be proved if we can show that the first zeros of $u^{\left(k^{\prime}\right)}$, $u^{\left(k^{\prime}+1\right)}, \ldots, u^{(n-1)}$ all coincide. Suppose then, to the contrary, that there exists an integer $r \in\left(k^{\prime}, n-1\right)$ such that $v^{(r)}\left(b_{r}\right)=0, v^{(n-1)}\left(b_{n-1}\right)=0$ and $b_{r} \in\left(0, b_{n-1}\right)$. The possibility $r=n-1$ may be excluded, since this would violate the extremal property of $v$. If $r$ is the largest integer with this property, we have $v^{(r+1)}\left(b_{r}\right) \neq 0$ and this shows that $v^{(r)}(t)$ changes its sign at the point $b_{r}$.

We now define a solution $V_{\epsilon}$ of (2.3) which has a zero of order $k^{\prime}$ at 0 and satisfies $V_{\epsilon}^{(\nu)}\left(b_{\nu}-\epsilon\right)=0, k^{\prime} \leqslant \nu \leqslant n-1, \nu \neq r, V_{\epsilon}^{\left(k^{\prime}\right)}(0)=v^{\left(k^{\prime}\right)}(0)$, where $\epsilon$ is a sufficiently small positive number. Because of the extremal property of $v$, $V_{\epsilon}$ is uniquely determined, and $V_{\epsilon} \rightarrow v$ for $\epsilon \rightarrow 0$. Since $v^{(r)}$ changes its sign at $b_{r}, V_{\epsilon}^{(r)}$ will necessarily have a zero in $\left(0, b_{n-1}-\epsilon\right)$ if $\epsilon$ is sufficiently small, and this conflicts with the extremal property of $v$. Hence, a point $b_{r} \in\left(0, b_{n-1}\right)$ such that $u^{(r)}\left(b_{r}\right)=0, r \in\left[k^{\prime}, n-1\right)$ cannot exist, and the zeros of $u^{\left(k^{\prime}\right)}, \ldots$, $u^{(n-1)}$ all coincide. This proves Theorem 2.1.

We now turn to Theorem 2.2. If $(2.1)$ is disconjugate on $[0, \infty)$, then the solution $u$ of (2.1) associated with the focal point $\zeta=\eta_{n-1,1}$ is positive on $(0, \infty)$, provided $u$ is normalized by $u^{(n-1)}(0)=1$. Hence $\left[u^{(n-1)}\right]^{\prime}=-p u<0$, and it follows from $u^{(n-1)}(\eta)=0$ that $u^{(n-1)}(c)<0$ for $c>\eta$. Since 


$$
\begin{aligned}
u(t)= & \sum_{\nu=0}^{n-2} \frac{u^{(\nu)}(c)}{\nu !}(t-c)^{\nu}+\frac{u^{(n-1)}(c)}{(n-1) !}(t-c)^{n-1} \\
& -\frac{1}{(n-1) !} \int_{c}^{t} p u(t-s)^{n-1} d s,
\end{aligned}
$$

this shows that $u(t)$ will become negative if $t$ is sufficiently large. Thus, $u(t)$ must have a zero in $(\eta, \infty)$ and this contradicts our assumption that $u$ is disconjugate.

We close this section with a remark concerning the applicability of our results to equations

$$
u^{(n)}+q(t) u=0
$$

whose coefficient functions $q$ are not assumed to be of constant sign. If we write $q_{+}=\max (q, 0)$ and $q_{-}=\min (q, 0)$, we have $q=q_{+}+q_{-}$and thus

$$
q_{-} \leqslant q \leqslant q_{+} \text {. }
$$

If $p_{1} \leqslant p_{2} \leqslant p_{3}$, the disconjugacy of $u^{(n)}+p_{1} u=0$ and $u^{(n)}+p_{3} u=0$ implies that of $u^{(n)}+p_{2} u=0$ [9], [13]. We may therefore conclude that conditions which guarantee the disfocality (and thus, by Theorem 2.1, the disconjugacy) of the equations $u^{(n)}+q_{+} u=0$ and $u^{(n)}+q_{-} u=0$ (which are of the types (2.1) and (2.2), respectively) also guarantee the disconjugacy of equation (2.5). An application of this remark will be made in $\$ 5$ (Theorem 5.5).

3. We begin by discussing the case of an $(n-1,1)$-focal point, in which the formalism is particularly simple. Since for an equation of the form $u^{(n)}-$ $p u=0, p>0$, such a point is excluded, it is sufficient to consider the equation

$$
u^{(n)}+p(t) u=0, \quad p(t)>0,
$$

where $p(t)$ is assumed to be continuous on $[0, \infty)$. If $u$ is taken to be the solution of (3.1) determined by the initial conditions $u^{(k)}(0)=0, k=0,1, \ldots$, $n-2, u^{(n-1)}(0)=1$, and we define the functions $\sigma_{k}$ by

$$
\sigma_{k}=u^{(k-1)} / u^{(n-1)},
$$

it follows by differentiation (and utilization of (3.1)), that the $\sigma_{k}$ satisfy the nonlinear differential system

$$
\sigma_{k}^{\prime}=\sigma_{k+1}+p \sigma_{1} \sigma_{k}, \quad \sigma_{k}(0)=0, k=1, \ldots, n-1\left(\sigma_{n} \equiv 1\right) .
$$

The initial conditions are a consequence of the fact that $u^{(n-1)}(0)=1$, while $u^{(k)}(0)=0$ for $k=1, \ldots, n-1$. Since the $(n-1,1)$-focal point of 0 coincides with the first zero of $u^{(n-1)}$ in $(0, \infty),(3.2)$ shows that the solutions of (3.3) will be continuous at least up to this focal point. On the other hand, not 
all the functions $\sigma_{k}$ can be continuous beyond this point. According to (3.2) this would imply that, in addition to $u^{(n-1)}$, all the functions $u, u^{\prime}, \ldots, u^{(n-2)}$ vanish at the focal point, i.e., $u$ would reduce to the trivial solution. We thus arrive at the following result.

THEOREM 3.1. In order that equation $(3.1)$ be $(n-1, n)$-disfocal on $[0, b)$, it is necessary and sufficient that the solution $\left(\sigma_{1}, \ldots, \sigma_{n-1}\right)$ of the nonlinear system (3.3) be continuous on $[0, b)$.

For $n=2,(3.3)$ reduces to the ordinary Riccati equation $\sigma^{\prime}=1+p \sigma^{2}$ $\left(\sigma=\sigma_{1}\right)$ with the initial condition $\sigma(0)=0$.

The usefulness of the system (3.3) for the treatment of our oscillation problem is due to the fact that the nonnegativity of the coefficients of the system results in certain positivity and monotonicity properties of its solutions. We shall later have occasion to apply similar arguments to more general systems, and we therefore state and prove the required results for systems which are sufficiently general to include all cases which will be encountered.

Lemma 3.1. Let $P_{r}\left(w_{1}, \ldots, w_{m}, t\right), r=1, \ldots, m$, be polynomials in the variables $w_{1}, \ldots, w_{m}$ whose coefficients are nonnegative and continuous functions on $[0, \infty)$. If the solutions $w_{1}, \ldots, w_{n}$ of the system of differential equations

$$
w_{r}^{\prime}=P_{r}\left(w_{1}, \ldots, w_{m}, t\right), \quad w_{r}(0)=0, \quad r=1, \ldots, m,
$$

are positive in some interval $(0, \epsilon], \epsilon>0$, then they remain positive throughout any interval $[0, b)$ in which all of them are continuous.

Lemma 3.2. Let $P_{r}\left(w_{1}, \ldots, w_{m}, t\right)$ and $P_{r}^{*}\left(w_{1}, \ldots, w_{m}, t\right), r=1, \ldots, m$, be two sets of polynomials which satisfy the hypotheses of Lemma 3.1. If, for positive $w_{1}, \ldots, w_{m}$ and all $t \in[0, b)$,

$$
P_{r}\left(w_{1}, \ldots, w_{m}, t\right) \leqslant P_{r}^{*}\left(w_{1}, \ldots, w_{m}, t\right) \quad(r=1, \ldots, m),
$$

and if there exist nonnegative differentiable functions $W_{1}, \ldots, w_{m}$ on $[0, b)$ which satisfy the conditions

$$
W_{r}^{\prime} \geqslant P_{r}^{*}\left(W_{1}, \ldots, W_{m}, t\right), \quad W_{r}(0)=0, \quad r=1, \ldots, m,
$$

then the differential system

$$
w_{r}^{\prime}=P_{r}\left(w_{1}, \ldots, w_{m}, t\right), \quad w_{r}(0)=0, \quad r=1, \ldots, m,
$$

has a continuous solution $\left(w_{1}, \ldots, w_{m}\right)$ on $[0, b)$, and $w_{r}(t) \leqslant w_{r}(t), r=1$, ..., $m$. 
Lemma 3.1 follows from the elementary observation (cf. [1, Chapter IV, $\S 8])$ that at the first point $t_{0}$ in $(\epsilon, b)$ at which one of the functions $w_{r}-$ say $w_{\rho}$-vanishes, we would have

$$
0=w_{\rho}\left(t_{0}\right)=w_{\rho}(\epsilon)+\int_{\epsilon}^{t_{0}} P_{\rho} d t \geqslant w_{\rho}(\epsilon)>0 .
$$

To prove Lemma 3.2, we solve the system (3.5) by the iteration procedure

$$
w_{r}^{(\nu+1)}(t)=\int_{0}^{t} P_{r}\left(w_{1}^{(\nu)}, \ldots, w_{m}^{(\nu)}, s\right) d s, \quad w_{r}^{(1)}(t)=w_{r}(t),
$$

where the $w_{r}$ are functions subject to (3.4). For $\nu=1$, we obtain

$$
w_{r}^{(2)}(t)=\int_{0}^{t} P_{r}\left(W_{1}, \ldots, w_{m}, t\right) d t, \quad t \in[0, b),
$$

and therefore, because of the nonnegativity of $w_{1}, \ldots, w_{m}$,

$$
0 \leqslant w_{r}^{(2)}(t) \leqslant \int_{0}^{t} P_{r}^{*}\left(w_{1}, \ldots, w_{m}, t\right) d t \leqslant w_{r}(t)=w_{r}^{(1)}(t) .
$$

Hence, $w_{r}^{(2)}(t) \leqslant w_{r}^{(1)}(t)$ for $r=1, \ldots, m$. Since the coefficients of the polynomial $P_{r}$ are nonnegative, the same is true of the coefficients of its multi-Taylor expansion in terms of the variables $w_{1}, \ldots, w_{m}$, and this implies that $P_{r}\left(w_{1}, \ldots, w_{m}, t\right) \leqslant P_{r}\left(w_{1}^{*}, \ldots, w_{m}^{*}, t\right)$ if $w_{1} \leqslant w_{1}^{*}, \ldots, w_{m} \leqslant w_{m}^{*}$. Accordingly, we can infer from

$$
\begin{aligned}
w_{r}^{(\nu+1)}(t) & -w_{r}^{(\nu)}(t) \\
& =\int_{0}^{t}\left[P_{r}\left(w_{1}^{(\nu)}, \ldots, w_{m}^{(\nu)}, s\right)-P_{r}\left(w_{1}^{(\nu-1)}, \ldots, w_{m}^{(\nu-1)}, s\right)\right] d s
\end{aligned}
$$

that $w_{r}^{(\nu+1)}(t) \leqslant w_{r}^{(\nu)}(t), r=1, \ldots, m$, provided that $w_{r}^{(\nu)}(t) \leqslant w_{r}^{(\nu-1)}(t), r=$ $1, \ldots, m$. It is also clear from (3.6) that the nonnegativity of the $w_{r}^{(\nu)}$ implies that of the $w_{r}^{(\nu+1)}$. Since, as shown above, $0 \leqslant w_{r}^{(2)}(t) \leqslant w_{r}^{(1)}(t)$, it follows that the sequences of continuous functions $w_{r}^{(1)}, w_{r}^{(2)}, \ldots$ decrease monotonically and are bounded below by zero. Hence, there exists a set of continuous limit functions $w_{1}, \ldots, w_{m}$, which is necessarily a set of solutions of the system (3.5) in $[0, b)$. Finally, the last assertion of Lemma 3.2 follows from the observation that $w_{r}(t) \leqslant w_{r}^{(1)}(t)=w_{r}(t)$.

Since the solution $u$ of (3.1) which was used in the definition (3.2) of $\sigma_{k}$ was defined by the initial conditions $u(0)=u^{\prime}(0)=\ldots=u^{(n-2)}(0)=0$, $u^{(n-1)}(0)=1$, all the functions (3.2) are positive in a small interval $(0, \epsilon], \epsilon>0$. We may therefore conclude from Lemma 3.1 that the solutions $\sigma_{k}$ of (3.3) (with the initial conditions $\left.\sigma_{k}(0)=0, k=1, \ldots, n-1\right)$ are positive as long as they are all continuous. If the function $p(t)$ is replaced by a function $q(t)$ such that 
$p(t) \leqslant q(t)$ on $[0, \infty)$, the coefficients of the system (3.3) do not decrease, and Lêmma 3.2 (with equality in (3.4)) becomes applicable. Because of Theorem 3.1, this leads to the following comparison theorem for equations of the type (3.1).

THEOREM 3.2. If $\eta(p)$ and $\eta(q)$ are the $(n-1,1)$-focal points associated with the differential equations $u^{(n)}+p u=0, v^{(n)}+q v=0,0<p \leqslant q$, respectively, then $\eta(q) \leqslant \eta(p)$.

We next show that Theorem 3.1 can be used to obtain explicit criteria for the absence of an $(n-1,1)$-focal point on a given interval. For simpler writing, we formulate these criteria for the interval $(0, \infty)$.

THEOREM 3.3. If equation (3.1) is disfocal, then

$$
t^{\nu} \int_{t}^{\infty} p(s) s^{n-1-v} d s \leqslant\left(\frac{n-1}{v}\right)(n-1) !
$$

for all $\nu \in(0, n-1]$ and all $t \in(0, \infty)$.

THEOREM 3.4. If there exists a function $R(t)$ of class $C^{n-1}(0, \infty)$ such that $R(t)=o\left(t^{n-2}\right)$ near $t=0, R>0$ and

$$
\int_{t}^{\infty} p R d s \leqslant R^{(n-1)}(t)
$$

for all $t \in(0, \infty)$, then equation (3.1) is disfocal.

The simplest choice for the function $R(t)$ in Theorem 3.4 is $R(t)=t^{n-1-\nu}$, where $\nu<1$. This leads to the following

COROLlARY. The conclusion of Theorem 3.4 holds if, for any $\nu<1$ and all $t \in(0, \infty)$,

$$
t^{\nu} \int_{t}^{\infty} p(s) s^{n-1-\nu} d s \leqslant \prod_{m=1}^{n-1}(n-\nu)
$$

The precision of (3.7) and (3.9) is indicated by the fact that, for $0<v<1$, the left-hand side of the necessary condition (3.7) and the sufficient condition (3.9) are identical, the two inequalities differing only in the value of the constant on the right-hand side. We also note that, for $\nu=0,(3.9)$ yields the known sufficient condition

$$
\int_{0}^{\infty} p(s) s^{n-1} d s \leqslant(n-1) !
$$

(cf. [4] for the case $n=2$, and [12] for the general case).

We first prove Theorem 3.3. Because of Theorem 3.1, our assumptions imply that the solution $\left(\sigma_{1}, \ldots, \sigma_{n-1}\right)$ of $(3.3)$ is continuous on $[0, \infty)$. For $p \equiv 0$, 
(3.3) has the solution $\sigma_{k}^{*}=[(n-k) !]^{-1} t^{n-k}, k=1, \ldots, n-1$. Hence, by Lemma 3.2, $\sigma_{k} \geqslant \sigma_{k}^{*}$, and therefore $\sigma_{k} \rightarrow \infty$ for $t \rightarrow \infty$. Moreover, since $\sigma_{2}>0$, (3.3) implies the inequality $\sigma_{1}^{\prime} \geqslant p \sigma_{1}^{2}$. Thus, for $0<\epsilon \leqslant 1$,

$$
\frac{1}{\epsilon \sigma_{1}^{\epsilon}}=\int_{t}^{\infty} \frac{\sigma_{1}^{\prime}}{\sigma_{1}^{1+\epsilon}} d s \geqslant \int_{t}^{\infty} p \sigma_{1}^{1-\epsilon} d s,
$$

i.e., because of $\sigma_{1} \geqslant[(n-1) !]^{-1} t^{n-1}$,

$$
\frac{1}{\epsilon} \geqslant \sigma_{1}^{\epsilon} \int_{t}^{\infty} p \sigma_{1}^{1-\epsilon} d s \geqslant \frac{t^{\epsilon(n-1)}}{(n-1) !} \int_{t}^{\infty} p s^{(n-1)(1-\epsilon)} d s .
$$

Setting $\nu=\epsilon(n-1)$, we obtain (3.7).

To prove Theorem 3.4, we choose a function $R(t)$ whose behavior near $t=0$ and whose differentiability properties are as indicated in the statement, and we define functions $\tau_{k}$ by

$$
\tau_{k}=R^{(k-1)} / \int_{t}^{\infty} p R d s, \quad k=1, \ldots, n-1 .
$$

Differentiating, we find that

$$
\begin{aligned}
\tau_{k}^{\prime} & =\tau_{k+1}+p \tau_{1} \tau_{k}, \quad k=1, \ldots, n-2, \\
\tau_{n-1}^{\prime} & =R^{(n-1)} / \int_{t}^{\infty} p R d s+p \tau_{1} \tau_{n-1},
\end{aligned}
$$

where, by (3.11) and the assumption $R(t)=o\left(t^{n-2}\right), \tau_{k}(0)=0, k=1, \ldots$, $n-1$. The differential system (3.12) is very similar to the system (3.3), the only difference being that the first term on the right-hand side of the last equation in (3.3) is 1 , rather than the expression appearing in (3.12). Since $\sigma_{k}(0)=\tau_{k}(0)=0$, $k=1, \ldots, n-1$, an application of Lemma 3.2 shows that the solutions of (3.3) will be continuous on $[0, \infty)$ if $\int_{t}^{\infty} p R d s \leqslant R^{(n-1)}(t)$ for all $t \in(0, \infty)$. The assertion of Theorem 3.4 now follows from Theorem 3.1.

4. The results of the preceding section can be extended to the case of a $(k, n-k)$-focal point, where $k \in[1, n-1]$ and is otherwise arbitrary. This will be done in $\S 5$. In the present section we consider an essentially different nonlinear approach to the problem of characterizing the presence or absence of an $(n-1,1)$-focal point. We shall prove

THEOREM 4.1. For equation (3.1) to be $(n-1,1)$-disfocal, it is necessary that the solution of

$$
T^{\prime}=1+p(t) T^{n} /(n-1)(n-1) !, \quad T(0)=0,
$$

be continuous on $[0, \infty)$, and sufficient that the solution of 


$$
T^{\prime}=1+p(t) T^{n} /(n-1) !, \quad T(0)=0,
$$

be continuous on this interval.

For $n=2$, the equations in both (4.1) and (4.2) coincide with the ordinary Riccati equation. For $n>2$, the two equations are different, and the question arises (but does not seem to be easy to answer) whether the numerical coefficients multiplying $p(t)$ in (4.1) and (4.2) are the best possible. It may be added here that, considering that these conditions refer to a phenomenon associated with an $n$th order equation, it does seem remarkable that the necessary and the sufficient conditions are both expressed in terms of first-order equations which differ only in the magnitude of a numerical coefficient.

The proof will be based on

LEMmA 4.1. If the solution $\left(\sigma_{1}, \ldots, \sigma_{n-1}\right)$ of the system (3.3) is continuous on $[0, b)$, and if we set

$$
(n-k) ! \sigma_{k}=\Phi_{k}^{n-k}, \quad k=1, \ldots, n-1, \quad \Phi_{k} \geqslant 0,
$$

then

$$
\Phi_{k} \leqslant \Phi_{k+1}, \quad k=1, \ldots, n-2, \quad t \in[0, b) .
$$

We note that, as shown before, $\sigma_{k}>0$ for $t>0$ and that therefore the extraction of the $(n-k)$ th root indicated in (4.3) is possible. We also remark that, as shown by (3.3), $(n-k) ! \sigma_{k}(t)=t^{n-k}+o\left(t^{n-k}\right)$ near $t=0$. It thus follows from the definition (4.3) that $\Phi_{k}=t+o(t)$ near $t=0$ for all $k$, which points up the precision of the inequalities (4.4).

(4.4) will be proved by an induction argument. Substituting (4.3) in (3.3) and simplifying, we obtain

$$
\Phi_{k}^{\prime}=\left(\frac{\Phi_{k+1}}{\Phi_{k}}\right)^{n-k-1}+\frac{S}{n-k} \Phi_{k}, \quad \Phi_{k}(0)=0, k=1, \ldots, n-1, \Phi_{n} \equiv 1,
$$

where $S$ is the expression

$$
S=p \Phi_{1}^{n-1} /(n-1) !
$$

By (4.5),

$$
\begin{aligned}
\left(\Phi_{k+1}-\Phi_{k}\right)^{\prime}= & \left(\frac{\Phi_{k+2}}{\Phi_{k+1}}\right)^{n-k}-\left(\frac{\Phi_{k+1}}{\Phi_{k}}\right)^{n-k-1} \\
& +S\left[\frac{\Phi_{k+1}-\Phi_{k}}{n-k}+\frac{. \Phi_{k}}{(n-k)(n-k-1)}\right]
\end{aligned}
$$


We wish to show that $\Phi_{k+1} \geqslant \Phi_{k}$, provided $\Phi_{k+2} \geqslant \Phi_{k+1}$. If the latter inequality holds, we have

$$
\left(\Phi_{k+1}-\Phi_{k}\right)^{\prime} \geqslant 1-\left(\frac{\Phi_{k+1}}{\Phi_{k}}\right)^{n-k-1}+\frac{S}{n-k}\left(\Phi_{k+1}-\Phi_{k}\right),
$$

since $\Phi_{k} \geqslant 0$, and by (4.6), $S \geqslant 0$. If our assertion is false, there must exist an interval $[\alpha, \beta)$ such that $\Phi_{k+1}-\Phi_{k}=0$ at $\alpha$ and $\Phi_{k+1}-\Phi_{k} \leqslant 0$ (but not identically zero) in ( $\alpha, \beta)$; this becomes evident if it is observed that $\Phi_{k+1}-\Phi_{k}=0$ at 0 . By (4.8) this would imply

$$
\left(\Phi_{k+1}-\Phi_{k}\right)^{\prime} \geqslant \frac{S}{n-k}\left(\Phi_{k+1}-\Phi_{k}\right)
$$

i.e., the function

$$
\left(\Phi_{k+1}-\Phi_{k}\right) \exp \left\{\frac{1}{n-k} \int_{\alpha}^{t} S d s\right\}
$$

would be nondecreasing in $[\alpha, \beta)$. Since this function vanishes at $\alpha$, the assumption that it is negative on some subinterval of $(\alpha, \beta)$ has thus led to a contradiction. Hence, $\Phi_{k+1} \geqslant \Phi_{k}$ throughout $[0, b)$ if $\Phi_{k+2} \geqslant \Phi_{k+1}$ in this interval.

To complete the proof of Lemma 4.1 we have to show that $\Phi_{n-1}-\Phi_{n-2}$ $\geqslant 0$. To do so, we note that, for $k=n-1,(4.5)$ takes the form

$$
\Phi_{n-1}^{\prime}=1+S \Phi_{n-1},
$$

and the corresponding inequality (4.7) is

$$
\left(\Phi_{n-1}-\Phi_{n-2}\right)^{\prime}=1-\left(\Phi_{n-1} / \Phi_{n-2}\right)+S\left(\Phi_{n-1}-\Phi_{n}\right) .
$$

But this is a special case of inequality (4.8) (except for the exponent $n-k-1$, which is irrelevant in this context), which was just shown to imply the inequality $\Phi_{k+1}-\Phi_{k} \geqslant 0$ throughout $[0, b)$. Hence, $\Phi_{n-1}-\Phi_{n-2} \geqslant 0$, and the proof is complete.

Turning now to the proof of Theorem 4.1, we apply assertion (4.4) of Lemma 4.1 to the case $k=1$. Because of (4.6), the first equation (4.5) is then found to imply the inequality

$$
\Phi_{1}^{\prime} \geqslant 1+p \Phi_{1}^{n} /(n-1)(n-1) !
$$

If equation (3.1) is $(n-1,1)$-disfocal, the solution $\left(\sigma_{1}, \ldots, \sigma_{n-1}\right)$ of $(3.3)$ is continuous throughout $[0, \infty)$ (by Theorem 3.1). By (4.3), the same is true of the functions $\Phi_{k}$. But $\Phi_{k}(0)=0$, and the function $\Phi_{1}$ is subject to the differential inequality (4.10). Comparing (4.10) with (4.1) and applying Lemma 3.2, we 
find that the solution of $(4.1)$ remains continuous throughout $[0, \infty)$. This proves the "necessary" part.

By (4.9) and (4.6),

$$
\Phi_{n-1}^{\prime}=1+p \Phi_{1}^{n-1} \Phi_{n-1} /(n-1) !
$$

and therefore, by (4.4),

$$
\Phi_{n-1}^{\prime} \leqslant 1+p \Phi_{n-1}^{n} /(n-1) !, \quad \Phi_{n-1}(0)=0 .
$$

By Lemma 3.2, $\Phi_{n-1}$ will thus be continuous in $[0, \infty)$ if the same is true of the function $T$ defined in (4.2). Because of (4.4) (and the fact that the $\Phi_{k}$ are positive and increasing as long as they are continuous), the functions $\Phi_{1}, \ldots, \Phi_{n-2}$ are also continuous on $[0, \infty)$. In view of $(4.3)$, the continuity of the solution $T$ of (4.2) thus guarantees that the solution of (3.3) remains continuous on $[0, \infty)$, and a reference to Theorem 3.1 completes the proof of Theorem 4.1.

5. In the present section we generalize the results of $\S 3$ to the general case of a $(k, n-k)$-focal point $(1 \leqslant k \leqslant n)$ for both the equations

$$
u^{(n)}+p u=0, \quad p>0
$$

and

$$
u^{(n)}-p u=0, \quad p>0 .
$$

We recall from $\S 2$ that equations (5.1) and (5.2) cannot have $(k, n-k)$-focal points for which $n-k$ is even or odd, respectively, and that it is therefore sufficient to consider the case of a $(k, n-k)$-focal point of the equation

$$
u^{(n)}-(-1)^{n-k} p u=0, \quad p>0 .
$$

We denote by $u_{\nu}$ the solution of (5.3) determined by the initial conditions

$$
u_{\nu}^{(\mu)}(0)=\delta_{\nu, \mu+1}, \quad \mu=0,1, \ldots, n-1,
$$

and we use the symbol $U_{\mu}$ for the $(n-k)$-dimensional row vector

$$
U_{\mu}=\left(u_{k+1}^{(\mu-1)}, \ldots, u_{n}^{(\mu-1)}\right) \text {. }
$$

Equation (5.3) (for $u_{k+1}, \ldots, u_{n}$ ) is then equivalent to the system

$$
\begin{aligned}
& U_{\mu}^{\prime}=U_{\mu+1}, \quad \mu=1, \ldots, k, \\
& U_{\mu}^{\prime}=-U_{\mu+1}, \quad \mu=k+1, \ldots, n-1, \\
& U_{n}^{\prime}=-p U_{1},
\end{aligned}
$$


and a $(k, n-k)$-focal point $\eta$ of $t=0$ is characterized by the condition

$$
\operatorname{det}\{B(\eta)\}=0,
$$

where $B$ is. the $(n-k) \times(n-k)$ matrix

$$
B=\left(\begin{array}{c}
U_{k+1} \\
\vdots \\
U_{n}
\end{array}\right)
$$

Indeed, because of (5.5), (5.7) is equivalent to the existence of a linear combination of $u_{k+1}, \ldots, u_{n}$ whose derivatives of order $k, k+1, \ldots, n-1$ vanish at $\eta$, and, by (5.4), the set of solutions of (5.3) which have a zero of order $k$ at $t=0$ is identical with the solution space of (5.3) spanned by $u_{k+1}, \ldots, u_{n}$.

Accordingly, the interval of $(k, n-k)$-disfocality of (5.3) coincides with the interval $[0, b)$ in which the matrix $(5.8)$ is nonsingular (it should be noted that, by $(5.4), B(0)$ is the $(n-k) \times(n-k)$ unit matrix). This fact, and the analogy with (3.2), suggest that a Riccati system associated with the $(k, n-k)$-disfocality of (5.3) may be obtained by introducing the vector variables

$$
S_{\mu}=U_{\mu} B^{-1}, \quad \mu=1, \ldots, k .
$$

We shall show that this is indeed the case. Since, by (5.8) and (5.6),

$$
B^{\prime}=\left(\begin{array}{c}
U_{k+2} \\
\vdots \\
U_{n} \\
p U_{1}
\end{array}\right)=-C B-p\left(\begin{array}{l}
0 \\
\vdots \\
0 \\
U_{1}
\end{array}\right) \text {. }
$$

where $C$ is the $(n-k) \times(n-k)$ matrix

$$
C=\left(\begin{array}{cccc}
0 & 1 & \cdot & 0 \\
& \ddots & \ddots \\
& 0 & \ddots & 1 \\
0 & \ddots & \ddots & 0
\end{array}\right),
$$

it follows from (5.9) that

$$
\begin{aligned}
S_{\mu}^{\prime} & =S_{\mu+1}-U_{\mu} B^{-1} B^{\prime} B^{-1} \\
& =S_{\mu+1}+S_{\mu}\left[C+p\left(\begin{array}{c}
0 \\
\vdots \\
U_{1}
\end{array}\right) B^{-1}\right], \quad S_{k+1}=U_{k+1} B^{-1}
\end{aligned}
$$


If $S_{\mu}^{(1)}, \ldots, S_{\mu}^{(n-k)}$ are the components of the row vector $S_{\mu}$, we have

$$
S_{\mu}\left(\begin{array}{c}
0 \\
\vdots \\
U_{1}
\end{array}\right) B^{-1}=S_{\mu}^{(n-k)} U_{1} B^{-1}=S_{\mu}^{(n-k)} S_{1},
$$

and the equations for $S_{\mu}$ take the form

$$
S_{\mu}^{\prime}=S_{\mu+1}+S_{\mu} C+p S_{\mu}^{(n-k)} S_{1}, \quad \mu=1, \ldots, k-1 .
$$

To obtain the equation for $\mu=k$, we observe that $c_{1} B=U_{k+1}$, where $c_{1}$ is the $(n-k)$-dimensional row vector

$$
c_{1}=(1,0, \ldots, 0) \text {. }
$$

Thus $S_{k+1}=U_{k+1} B^{-1}=c_{1}$. Noting that, by (5.9), (5.4) and (5.5), the $S_{\mu}$ satisfy the initial conditions $S_{\mu}(0)=0$, we find that on an interval $[0, b)$ on which (5.3) is $(k, n-k)$-disfocal, the differential system

$$
S_{\mu}^{\prime}=S_{\mu+1}+S_{\mu} C+p S_{\mu}^{(n-k)} S_{1}, \quad \mu=1, \ldots, k\left(S_{\mu+1}=c_{1}\right), S_{\mu}(0)=0,
$$

has a continuous solution $\left(S_{1}, \ldots, S_{k}\right)$.

In order to prove that, conversely, the existence of such a solution implies the disfocality of (5.3), we have to show that (5.7) cannot hold as long as all the $S_{\mu}(\mu=1, \ldots, k)$ are continuous.

By (5.9), $S_{\mu} B=U_{\mu}$ and therefore, by (5.8), $U_{\mu}=\Sigma_{m=1}^{n-k} S_{\mu}^{(m)} U_{k+m}$ for $\mu=1, \ldots, k$, i.e., $U_{1}, \ldots, U_{n}$ are contained in the space $V$ spanned by $U_{k+1}$, $\ldots, U_{n}$. Suppose now that, for $t \rightarrow t_{0}, B$ becomes singular and the $S_{\mu}$ remain continuous. By (5.8), $V$ will be of dimension $\left\langle n-k\right.$ for $t=t_{0}$. Hence, there exists an $(n-k)$-dimensional nonzero constant vector $\alpha=\left(\alpha_{1}, \ldots, \alpha_{n-k}\right)$ such that $\alpha U_{r}\left(t_{0}\right)=0, r=1, \ldots, n$. Since $\alpha U_{r}\left(t_{0}\right)=u^{(r-1)}\left(t_{0}\right)$, where $u(t)=$ $\alpha_{1} u_{k+1}(t)+\ldots+\alpha_{n-k} u_{n}(t)$ is a solution of (5.3), it follows that $u(t)$ must be the trivial solution $u \equiv 0$. However, because of (5.4) and (5.5) (and the fact that $\alpha \neq 0$ ), this is impossible. This contradiction shows that $B$ remains nonsingular (and, thus, (5.3) remains ( $k, n-k)$-disfocal) as long as (5.12) has a continuous solution. This completes the proof of the following generalization of Theorem 3.1.

TheOREM 5.1. Let $C$ and $c_{1}$ denote, respectively, the $(n-k) \times(n-k)$ matrix (5.10) and the $(n-k)$-dimensional row vector (5.11). In order that equation $(5.3)$ be $(k, n-k)$-disfocal on an interval $[0, b)$, it is necessary and sufficient that the solution of the differential system (5.12) for the row vectors $S_{\mu}=\left(S_{\mu}^{(1)}\right.$, $\left.\ldots, S_{\mu}^{(n-k)}\right)$ be continuous on $[0, b)$. 
(5.12) may be rewritten as a system of scalar differential equations for the components of the vectors $S_{\mu}$. Since $p>0$ and the elements of $C$ and $c_{1}$ are nonnegative, all the coefficients of this system are nonnegative. Moreover, all the components of the $S_{\mu}$ are positive for $t \in(0, \epsilon]$ with sufficiently small $\epsilon$. Indeed, because of the initial conditions (5.4), all the components of the vector (5.5) are positive for $t \in(0, \epsilon]$, and the matrix $B^{-1}$ used in (5.9) reduces to the unit matrix for $t=0$. We may therefore conclude from Lemma 3.1 that all the components of $S_{1}, \ldots, S_{k}$ remain positive as long as the solution of (5.12) is continuous. Hence, Lemma 3.2 may be applied to the scalar differential systems equivalent to (5.12) for different coefficient functions $p$ and $q, p \leqslant q$. In conjunction with Theorem 5.1, this leads to the following generalization of Theorem 3.2.

THEOREM 5.2. If $0 \leqslant p(t) \leqslant q(t)$, and we denote by $\eta_{k, n-k}(p)$ and $\eta_{k, n-k}(q)$ the $(k, n-k)$-focal points associated with the differential equations $u^{(n)}-(-1)^{n-k} p u=0$ and $u^{(n)}-(-1)^{n-k} q u=0$, respectively, then $\eta_{k, n-k}(q)$ $\leqslant \eta_{k, n-k}(p)$.

We now turn to the problem of obtaining explicit criteria for the presence or absence of $(k, n-k)$-focal points. We begin with the analogue of Theorem 3.3.

THEOREM 5.3. If equation (5.3) is $(k, n-k)$-disfocal, then

$$
t^{\nu} \int_{t}^{\infty} p(s) s^{n-1-\nu} d s \leqslant \frac{(n-1)^{2}}{\nu}(k-1) !(n-k-1) !
$$

for all $\nu \in(0, n-1]$ and all $t \in(0, \infty)$.

It is of interest to note that the constant in (5.13) is not larger than the constant in (3.7). Hence, (3.7) holds if (5.3) is $(k, n-k)$-disfocal for any $k \in$ $[1, n-1]$, i.e., (3.7) is a necessary condition for the disfocality of both (5.1) and (5.2).

Proof. We again use the fact that all the coefficients appearing in (5.12) are nonnegative. Since, as pointed out above, all the components of the $S_{\mu}$ are positive as long as the solution of (5.12) is continuous, the assumption of $(k, n-k)$ disfocality (and the use of Theorem 5.1) leads to the conclusion

$$
\left[S_{1}^{(n-k)}\right]^{\prime} \geqslant p\left[S_{1}^{(n-k)}\right]^{2}
$$

from the first equation (5.12). To utilize this inequality, we further require a positive lower bound for $S_{1}^{(n-k)}$. By Lemma 3.2, lower bounds for the components of the solution vectors of (5.12) are provided by the corresponding components of the solution vectors of the system

$$
R_{\mu}^{\prime}=R_{\mu+1}+R_{\mu} C, \quad \mu=1, \ldots, k\left(R_{k+1}=c_{1}\right), R_{\mu}(0)=0 .
$$

These equations are equivalent to 


$$
\left(R_{\mu} e^{-C t}\right)^{\prime}=R_{\mu+1} e^{-C t}, \quad R_{k+1} e^{-C t}=c_{1} e^{-C t} .
$$

Thus, if we set $V_{\mu}=R_{\mu} e^{-C t}$, the system reduces to $V_{\mu}^{\prime}=V_{\mu+1}, V_{k+1}=c_{1} e^{-C t}$. Hence $V_{1}^{(k)}=c_{1} e^{-C t}$ and therefore, because of the initial conditions,

$$
R_{1} e^{-C t}=V_{1}=\frac{1}{(k-1) !} \int_{0}^{t}(t-s)^{k-1} c_{1} e^{-C s} d s,
$$

i.e.,

$$
R_{1}=\frac{1}{(k-1) !} \int_{0}^{t}(t-s)^{k-1} c_{1} e^{C(t-s)} d s
$$

Since

$$
c_{1} e^{C(t-s)}=\left(1, t-s, \frac{(t-s)^{2}}{2 !}, \ldots, \frac{(t-s)^{n-k-1}}{(n-k-1) !}\right.
$$

it follows that

$$
\psi \equiv S_{1}^{(n-k)} \geqslant R_{1}^{(n-k)}=\frac{t^{n-1}}{(k-1) !(n-k+1) !(n-1)} .
$$

This shows that $\psi \rightarrow \infty$ for $t \rightarrow \infty$. Since, by (5.14), $\psi^{\prime} \psi^{-1-\epsilon} \geqslant p \psi^{1-\epsilon}$, we thus have

$$
\psi^{\epsilon} \int_{t}^{\infty} p \psi^{1-\epsilon} d s \geqslant \epsilon
$$

for $\epsilon \in(0,1)$ and all positive $t$. Estimating $\psi$ by means of $(5.15)$ and setting $\epsilon(n-1)=\nu$, we obtain (5.13).

To obtain a sufficient condition for $(k, n-k)$-disfocality, we consider the vector functions $T_{\mu}=\left(T_{\mu}^{(1)}, \ldots, T_{\mu}^{(n-k)}\right), \mu=1, \ldots, k$, defined by

$$
T_{\mu}=\frac{R^{(\mu-1)}(n-k-1) ! Q}{\int_{t}^{\infty} s^{n-k-1} p R d s}
$$

where $Q$ is the row vector

$$
Q=\left(1, t, t^{2} / 2 !, \ldots, t^{n-k-1} /(n-k-1) !\right)
$$

and $R(t)$ is positive and of class $C^{k}$ on $(0, \infty)$ and satisfies the initial conditions $R(0)=R^{\prime}(0)=\ldots=R^{(k-1)}(0)=0$. Evidently, $Q^{\prime}=Q C$, where $C$ is the matrix (5.10). Hence, differentiating (5.16) and observing that $t^{n-k-1}=(n-k-1) ! Q^{(n-k)}$, we obtain

$$
T_{\mu}^{\prime}=T_{\mu+1}+T_{\mu} C+p T_{\mu}^{(n-k)} T_{1}, \quad \mu=1, \ldots, n-k .
$$

For $\mu=1, \ldots, k-1$, these equations are identical with the corresponding equations (5.12). For $\mu=k$ the first term on the right-hand side is $T_{k+1}$, while 
in (5.12) it is $c_{1}=(1,0, \ldots, 0)$. Accordingly, the coefficients of the system (5.12) will be dominated by those of the system (5.18) if the components of $T_{k+1}$ are not smaller than the corresponding components of $c_{1}$. By (5.16) and (5.17) this holds trivially for $T_{k+1}^{(2)}, \ldots, T_{k+1}^{(n-k)}$, and for the first components this will be assured by the condition

$$
\frac{1}{(n-k-1) !} \int_{t}^{\infty} s^{n-k-1} p R d s \leqslant R^{(k)}(t), \quad t \in(0, \infty) .
$$

The solution vectors $S_{\mu}$ of (5.12) satisfy the initial conditions $S_{\mu}(0)=0, \mu=1$, $\ldots, k$. By (5.16) and the initial conditions imposed on $R$, we also have $T_{\mu}(0)=$ $0, \mu=1, \ldots, k$. Applying Lemma 3.2, we therefore obtain

THEOREM 5.4. Equation (5.3) will be $(k, n-k)$-disfocal on $[0, \infty)$ if there exists a function $R(t)$ with the following properties: (a) $R(t) \in C^{k}(0, \infty)$; (b) $R(t) \geqslant 0$; (c) near $t=0, R(t)=o\left(t^{k-1}\right)$; (d) for all $t \in(0, \infty), R(t)$ satisfies the inequality (5.19).

The function $R(t)=t^{k-\nu}, \nu<1$, satisfies assumptions (a), (b), (c). Hence, we have the following

COROLlary. If

$$
t^{\nu} \int_{t}^{\infty} p s^{n-1-\nu} d s \leqslant(n-k-1) ! \prod_{m=1}^{k}(m-v)
$$

for some $\nu<1$ and all $t \in(0, \infty)$, equation (5.3) is $(k, n-k)$-disfocal on $[0, \infty)$.

As already remarked in connection with the special case (3.9) of (5.20), the precision of both the necessary condition (5.13) and the sufficient condition (5.20) is indicated by the fact that these two conditions differ only in the value of the constant on the right-hand side.

If we set

$$
M(\nu)=\min _{1 \leqslant k \leqslant n-1}(n-k-1) ! \prod_{m=1}^{k}(m-\nu), \quad \nu<1,
$$

the conditions (5.20) will be satisfied for all $k \in[1, n-1]$ if $t^{\nu} \int_{t}^{\infty} p s^{n-1-\nu} d s$ $\leqslant M(\nu)$ for all $t>0$ and some $\nu<1$. Accordingly, the latter condition guarantees the disfocality (and therefore, by Theorem 2.1, also the disconjugacy) of both equations (5.1) and (5.2). If we combine this with the remark made at the end of $\S 2$, and observe that $-|q| \leqslant q_{-} \leqslant q \leqslant q_{+} \leqslant|q|$, we obtain a disconjugacy criterion for the differential equation

$$
u^{(n)}+q(t) u=0
$$

which does not depend on the constancy of the sign of $q(t)$. 
THEOREM 5.5. Let $q(t)$ be continuous on $[0, \infty)$. If $t^{\nu} \int_{t}^{\infty}|q| s^{n-1-\nu} d s \leqslant$ $M(\nu)$ for all $t>0$ and some $\nu<1$, where $M(\nu)$ is the constant (5.21), then equation $(5.22)$ is disconjugate on $[0, \infty)$.

Since $M(0)=(n-2)$ ! we obtain, in particular, the sufficient disconjugacy criterion

$$
\int_{0}^{\infty}|q| s^{n-1} d s \leqslant(n-2) !
$$

(cf. [7]).

As a last application of Theorcm 5.1, we prove

THEOREM 5.6. If the differential equations $u^{(n)}-(-1)^{n-k} p_{r} u=0, p_{r}>$ $0, r=1, \ldots, N$ are $(k, n-k)$-disfocal on $[0, b)$, and if

$$
g(t)=\prod_{r=1}^{N}\left[p_{r}(t)\right]^{\alpha},
$$

where

$$
\alpha_{1}+\ldots+\alpha_{N}=1, \quad \alpha_{r}>0,
$$

then the equation $u^{(n)}-(-1)^{n-k} g u=0$ is also $(k, n-k)$-disfocal on $[0, b)$.

In the special case, $n=2, k=1$, this result is known (at least by word of mouth), and it is easily proved by utilizing properties of the associated selfadjoint eigenvalue problem $u^{\prime \prime}+\lambda p u=0, u(0)=u^{\prime}(b)=0$. However, this method cannot be extended to the general case.

To prove Theorem 5.6, we replace the system (5.12) associated with $u^{(n)}-$ $(-1)^{n-k} p_{r} u=0$ by a system of scalar differential equations

$$
\left[T_{\nu}^{(r)}\right]^{\prime}=P_{\nu}^{(r)}\left(T_{1}^{(r)}, \ldots, T_{m}^{(r)}, t\right), \quad \nu=1, \ldots, m,
$$

where $m=k(n-k)$. The polynomials $P_{\nu}^{(r)}$ can be written in the form

$$
P_{\nu}^{(r)}\left(T_{1}^{(r)}, \ldots, T_{m}^{(r)}, t\right)=\sum_{s} M_{s}\left(T_{1}^{(r)}, \ldots, T_{m}^{(r)}\right) f_{s}^{(r)}(t),
$$

where the $M_{s}$ are monomials and $f_{s}^{(r)}(t)$ is either a nonnegative constant independent of $r$, or else $f_{s}^{(r)}(t)=p_{r}(t)$. Because of Theorem 5.1, our assumptions imply that the $T_{1}^{(r)}, \ldots, T_{m}^{(r)}$ are continuous (and therefore, as shown before, also nonnegative) on $[0, b)$. We may therefore define positive functions $T_{1}, \ldots, T_{m}$ by

$$
T_{\nu}=\prod_{r=1}^{N}\left[T_{\nu}^{(r)}\right]^{\alpha}
$$

where the $\alpha_{r}$ are subject to (5.24). By (5.25) and (5.26), we then have 


$$
\frac{T_{\nu}^{\prime}}{T_{\nu}}=\sum_{s}\left(\sum_{r=1}^{N} \alpha_{r} \frac{M_{s}\left(T_{1}^{(r)}, \ldots, T_{m}^{(r)}\right)}{T_{\nu}^{(r)}} f_{s}^{(r)}(t)\right) .
$$

Since $M_{s}$ is a monomial and $f_{s}^{(r)}(t)$ has the properties indicated above, an application of the arithmetic-geometric inequality [1] yields

$$
\frac{T_{\nu}^{\prime}}{T_{\nu}} \geqslant \sum_{s} \frac{M_{s}\left(T_{1}, \ldots, T_{m}\right)}{T_{\nu}} \prod_{r=1}^{N}\left[f_{s}^{(r)}(t)\right]^{\alpha_{r}},
$$

if the right-hand side is simplified with the help of (5.27). Every one of the products appearing in this inequality has either the same constant value as all its factors, or else it is equal to the function (5.23) if $f_{s}^{(r)}(t)=p_{r}(t)$. In view of (5.26), the inequality thus reduces to

$$
T_{\nu}^{\prime} \geqslant P_{\nu}\left(T_{1}, \ldots, T_{m}, t\right),
$$

where $P_{\nu}$ is the polynomial obtained from $P_{\nu}^{(r)}$ if $P_{r}$ is replaced by $g$ in the original system (5.12).

Applying now Lemma 3.2, we find that the system of scalar equations

$$
\tau_{\nu}^{\prime}=P_{\nu}\left(\tau_{1}, \ldots, \tau_{m}, t\right), \quad \tau_{\nu}(0)=0,
$$

has a continuous solution on $[0, b)$. Since this system is equivalent to the system (5.12) (with $p$ replaced by the function (5.23)), an application of Theorem 5.1 completes the proof of Theorem 5.6.

\section{REFERENCES}

1. E. F. Beckenbach and R. Bellman, Inequalities, Ergebnisse der Mathematik und ihrer Grenzgebiete, Band 30, Springer-Verlag, Berlin, 1961. MR 28 \#1266.

2. W. A. Coppel, Disconjugacy, Lecture Notes in Math., vol. 220, Springer-Verlag, New York, 1971.

3. M. Hanan, Oscillation criteria for third-order linear differential equations, Pacific $J$. Math. 11 (1961), 919-944. MR 26 \#2695. MR 10, 376.

4. E. Hille, Non-oscillation theorems, Trans. Amer. Math. Soc. 64 (1948), 234-252.

5. H. C. Howard, Oscillation criteria for fourth-order linear differential equations, Trans. Amer. Math. Soc. 96 (1960), 296-311. MR 22 \#159.

6. W. J. Kim, On the zeros of solutions of $y^{(n)}+p y=0$, J. Math. Anal. Appl. 25 (1969), 189-208. MR 40 \#451.

7. V. A. Kondrat'ev, Oscillatory properties of solutions of the equation $y^{(n)}+p(x) y$ $=0$, Trudy Moskov. Mat. Obšč. 10 (1961), 419-436. (Russian) MR 25 \#5239.

8. W. Leighton and Z. Nehari, On the oscillation of solutions of self-adjoint linear differential equation of the fourth order, Trans. Amer. Math. Soc. 89 (1958), 325-377. MR 21 \#1429.

9. A. Ju. Levin, Some problems on the oscillation of solutions of linear differential equations, Dokl. Akad. Nauk SSSR 148 (1963), 512-515 = Soviet Math. Dokl. 4 (1963), 121-124. MR 26 \#3927. 
10. A. Ju. Levin, Distribution of the zeros of solutions of a linear differential equation, Dokl. Akad. Nauk SSSR 156 (1964), 1281-1284 = Soviet Math. Dokl. 5 (1964), 818-821. MR 29 \#1378.

11. The nonoscillation of solutions of the equation $X^{(n)}+p_{1}(t) X^{(n-1)} \times \ldots$ $\times p_{n}(t) X=0$, Uspehi Mat. Nauk 24 (1969), no. 2 (146), 43-96 = Russian Math. Surveys 24 (1969), no. 2, 43-99. MR 40 \#7537.

12. Z. Nehari, Non-oscillation criteria for $n$-th order linear differential equations, Duke Math. J. 32 (1965), 607-615. MR 32 \#338.

13. - Disconjugate linear differential operators, Trans. Amer. Math. Soc. 129 (1967), 500-516. MR 36 \#2860.

14. - Conjugate points, triangular matrices, and Riccati equations, Trans. Amer. Math. Soc. 199 (1974), 181-198.

15. W. T. Reid, Ordinary differential equations, Wiley, New York, 1971. MR 42 \#7963.

16. T. L. Sherman, Properties of solutions of nth order linear differential equations, Pacific J. Math. 15 (1965), 1045-1060. MR 32 \#2654.

17. C. A. Swanson, Comparison and oscillation theory of linear differential equations, Academic Press, New York, 1968.

DEPARTMENT OF MATHEMATICS, CARNEGIE-MELLON UNIVERSITY, PITTSBURGH, PENNSYLVANIA 15213 